\title{
A CHARACTERIZATION OF B*-ALGEBRAS
}

\author{
A. K. GAUR \\ Department of Mathematics \\ Duquesne University, \\ Pittsburgh, PA 15282
}

(Received December 11, 1995 and in revised form August 16, 1996)

\begin{abstract}
A characterization of $B^{*}$-algebras amongst all Banach algebras with bounded approximate identities is obtained.
\end{abstract}

1991 AMS Subject Classification: 46J99, 46J15

Key Words and Phrases: Approximate identity; $B^{*}$-algebra; self-adjoint elements;

Hermitian elements.

\section{Introduction.}

We recall that an approximate identity in a Banach algebra $A$ is a net $\left\{e_{\alpha}: \alpha \in I\right\}$ in $A$ where $I$ is a directed set such that $\lim _{\alpha} e_{\alpha} x=x=\lim _{\alpha} x e_{\alpha}$ for every $x$ in $A$. If there is a finite constant $M$ such that $\left\|e_{\alpha}\right\| \leq M$ for all $\alpha$, then the approximate identity is said to be bounded.

Let $A$ be a Banach algebra. For each $x$ in $A$, let

$$
D_{A}(x)=\left\{f \in A^{\prime}:\|f\|=1=f(x)\right\} .
$$

By a corollary of the Hahn-Banach theorem, $D_{A}(x)$ is non-empty. We denote $S(A)=\{x \in A$ : $\|x\|=1\}$.

For each $a \in A$, we call the set $V_{A}(a)=\left\{f(a x): f \in D_{A}(x), x \in S(A)\right\}$ the spatial numencal range of $a$.

We recall [5] that the relative numerical range of $a$ in $A$ with respect to $x \in A$, is defined as

$$
\stackrel{\circ}{V}_{x}(A, a)=\left\{f(a x): f \in D_{A}(x)\right\} .
$$

Thus we see that $V_{A}(a)=\bigcup\left\{\stackrel{\circ}{V}_{x}(A, a): x \in S(A)\right\}$, which is a bounded subset of the complex numbers bounded by $\|a\|$.

If $A$ has an approximate identiiy of norm less than or equal to one then $A$ can embedded, isometrically and isomorphically, in a unital Banach algebra $A^{+}$in such a way that for each $a$ in $A$

$$
V\left(A^{+}, a\right)=\overline{c o} V_{A}(a)
$$

where $V\left(A^{+}, a\right)=\left\{f(a): f \in\left(A^{+}\right)^{\prime},\|f\|=1=f(a)=\|a\|\right\}$. For details see [4], Theorem 2.3. 
An element $h$ of a Banach algebra $A$ is said to be Hermitian if $V_{A}(a) \subset R$. We denote by $H(A)$ the set of all Hermitian elements of $A$. A $B^{*}$-algebra is a Banach algebra $A$ with an involution, $a \rightarrow a^{*}$ satisfying the following conditions:

(1) $(a+b)^{*}=a^{*}+b^{*}$;

(2) $(a b)^{*}=b^{*} a^{*}$;

(3) $(\alpha a)^{*}=\bar{\alpha} a^{*}$;

(4) $a^{* *}=a$; and

(5) $\left|a^{*} a\right|=|a|^{2}$

for all $a, b$ in $A$ and $\alpha$ in $C$.

An element $a$ in a $B^{*}$-algebra is said to be self-adjoint if $a=a^{*}$. The set of all self adjoint elements will be denoted by $S(A)$. Each element $a \in A$ can be written uniquely in the form $a=h+i k$ where $h, k \in S(A)$. Some of the well known properties of $S(A)$ are the following:

a) The set $S(A)$ is a real partially ordered Banach space,

b) each of its elements has real spectrum,

c) if $h, k \in S(A)$ then $i(h k-k h) \in S(A)$, and

d) for each $h \in S(A)$, the spectral radius $\rho(h)=\|h\|$.

It is clear that the set of Hermitian elements, $H(A)$, of a Banach algebra with a bounded approximate identity of norm less than or equal to one has many of the properties of $S(A)$ in a $B^{*}$-algebra.

In this note we prove that in an arbitrary $B^{*}$-algebra $A, H(A)=S(A)$ in Theorem 2.1. This results mimics a result by Bohnenblust and Karlin [2].

In [8], Vidav has shown that a unital Banach algebra $A$ with the following conditions:

(1) $A=H(A)+i H(A)$;

(2) for each $h$ in $H(A)$ there exists $h_{1}, h_{2}$ in $H(A)$ such that $h_{1}+i h_{2}=h^{2}$ and $h_{1} h_{2}=h_{2} h_{1}$

is a $B^{*}$-algebra with Vidav-involution. Combining the results of Vidav [8], Berkson [1], and Glickfeld [6] we obtain the result that if $A$ is a unital Banach algebra such that $A=H(A)+i H(A)$ then $A$ is a $B^{*}$-algebra under the Vidav-involution. Here, we extend this result to the nonunital case in the form of Lemma 3.1.

Finally, combining the results of Theorem 2.1 and Lemma 3.1 we have a characterization of $B^{*}$-algebras with bounded approximate identities.

\section{Some Results.}

We now prove the following theorem.

Theorem 2.1 Let $A$ be a $B^{*}$-algebra with a bounded approximate identity of norm less than or equal to one. An element of $A$ is Hermitian if and only if it is self-adjoint.

Proof. Case 1. Suppose that $A$ has a unit element 1. Let $f \in D_{A}(1)$. Then it is known that such a functional has the property that $f\left(h^{*}\right)=\overline{f(h)}$, for every $h$ in $A$. Thus if $h$ is a self-adjoint element of $A, f(h)=f\left(h^{*}\right)=\overline{f(h)}$ and hence $f(h)$ is real for all $f$ in $D_{A}(1)$. Hence, $S(A) \subseteq H(A)$.

Case 2. If $A$ has no identity element then it will have an approximate identity of norm less than or equal to one. Also, with the involution defined by $(a, \alpha)^{*}=\left(a^{*}, \bar{\alpha}\right)$ for $(a, \alpha) \in A^{+}$, and 
by Theorem 2.3 in [4], $A^{+}$becomes a unital $B^{*}$ - algebra containing as a sub- $B^{*}$-algebra, ([3], 1.3.8).

Let $h$ be a self-adjoint element of $A$. Then $(h, 0)$ is self-adjoint and hence Hermitian in the unital $B^{*}$-algebra $A^{+}$. Hence $h \in H(A)$. We have therefore for any $B^{*}$-algebra, $S(A) \subseteq H(A)$.

Suppose conversely that $h \in H(A)$. Then for $h_{1}$ and $h_{2}$ in $S(A), h=h_{1}+i h_{2}$. This implies that $\nu\left(h_{2}\right)=0$ (where $\nu(x)=\sup \left\{|\lambda|: \lambda \in V_{A}(x)\right\}$ and is called numerical radius of $x$ in $A$ ) and hence $h_{2}=0$. Thus $h=h_{1}$ so that $h$ is self-adjoint. That is $H(A) \subseteq S(A)$ and hence the theorem.

Remark 2.1 The above theorem shows that in a $B^{*}$-algebra the Hermitian elements generate the whole algebra in the sense that each element $a$ may be written in the form $a=h_{1}+i h_{2}$ with $h_{1}$ and $h_{2}$ in $H(A)$. In an arbitrary Banach algebra $A$ this is not true. We therefore consider the set $J(A)=H(A)+i H(A)$. Since $H(A)$ is a real space it follows that $J(A)$ is a complex linear space. If $A$ has no unit element then by Theorem 2.3, [4], $J(A) \times C=J\left(A^{+}\right)$. We define a map $a \rightarrow a^{*}$ from $J(A)$ into itself by

$$
\left(h_{1}+i h_{2}\right)^{*}=h_{1}-i h_{2}, \text { for all } h_{1}, h_{2} \in H(A) .
$$

The linear map $a \rightarrow a^{*}$ is known as the Vidav-involution on $J(A)$.

Remark 2.2 If $A$ has no unit element then it is a simple matter to verify that the Vidavinvolution on $J\left(A^{+}\right)$is an extension of the Vidav-involution on $J(A)$. The space $J(A)$ is a complex Banach space and $a \rightarrow a^{*}$ is a continuous linear involution on $J(A)$. In general, the Banach space $J(A)$ is not an algebra, and if $J(A)$ is an algebra under some conditions, then the Vidav-involution has the additional property

$$
(a b)^{*}=a^{*} b^{*}, \text { for all } a, b \in J(A) .
$$

\section{Characterization.}

Vidav has shown in [8] that a unital Banach algebra $A$ with the following conditions:

(V1) $A=H(A)+i H(A)$,

(V2) for each $h$ in $H(A)$ there exists $h_{1}, h_{2}$ in $H(A)$ such that $h_{1}+i h_{2}=h^{2}$ and $h_{1} h_{2}=h_{2} h_{1}$, is a $B^{*}$-algebra with Vidav-involution and a norm equivalent to the original norm on $A$.

According to Palmer [7], the condition (V1) implies (V2). Also Berkson [1], Glickfeld [6], and Palmer [7] have shown that if (V1) is satisfied by the algebra $A$ the equivalent norm by Vidav is equal to the original norm on $A$. So by these results we have the result that if $A$ is a unital Banach algebra satisfying (V1) then $A$ is $B^{*}$-algebra under the Vidav-involution. The following lemma extends this result to the non-unital case.

Lemma 3.1 Let $A$ be a Banach algebra with a bounded approximate identity of norm less than or equal to one. Suppose that every $a$ in $A$ has the form $a=h_{1}+i h_{2}$, for all $h_{1}, h_{2}$ in $H(A)$. Then with the Vidav-involution, $A$ is a $B^{*}$-algebra.

Proof. From Remark 2.1 we have that $J\left(A^{+}\right)=J(A) \times C$. Since $J(A)=A$ (by the hypothesis) we have $J\left(A^{+}\right)=A^{+}$. Therefore $A^{+}$is a unital $B^{*}$-algebra under the Vidavinvolution. Furthermore, $A$ is a closed and self adjoint subalgebra of $A^{+}$, and is therefore a $B^{*}$-algebra under the Vidav-involution. 
Finally, combining the results of Theorem 2.1 and Lemma 3.1 we have the following:

Theorem 3.2 Let $A$ be a Banach algebra with a bounded approximate identity of norm less than or equal to one. Then $A$ is a $B^{*}$-algebra under some involution if and only if each element $a$ of $A$ can be written in the form $a=h_{1}+i h_{2}$ where $h_{1}$ and $h_{2}$ are Hermitian elements of $A$.

\section{Acknowledgement.}

The author expresses his appreciation to the referee for his or her valuable suggestions which improved the clarity of this presentation.

\section{Ref e r e n c e s}

[1] E. Berkson, "Some characterizations of $C^{*}$-algebras", Mlinois J. Math., 10, (1966), 1-8.

[2] H.F. Bohnenblust and S. Karlin, "Geometrical properties of the unit sphere of Banach algebras", Ann. of Math, 62, (1955), 217-229.

[3] J. Dixmier, "Les $C^{*}$-algébres et leurs représentations", Gauthier Villars, 1964.

[4] A.K. Gaur and T. Husain, "Spatial numerical ranges of elements of Banach algebras", Internat. J. Math. and Math. Sci., 12, (1989), 633-640.

[5] A.K. Gaur and T. Husain, "Relative numerical ranges", Math. Japonica, 36, (1991), 127135.

[6] B.W. Glickfeld, "A metric characterization of $C(X)$ and its generalization to $C^{*}$-algebras", Illinois J. of Math., 10, (1966), 547-566.

[7] T.W. Palmer, "Characterization of $C^{*}$-algebras", Bull. Amer. Math. Soc., 74, (1968), 538-540.

[8] I. Vidav, "Eine metrische kennzeichnung der selbstad jungierten operatoren", Math. Z., 66, (1956), 121-128. 


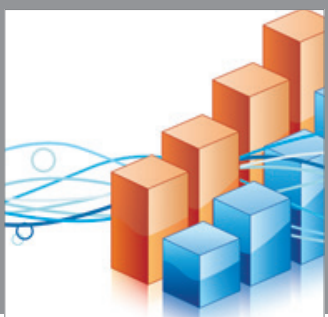

Advances in

Operations Research

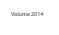

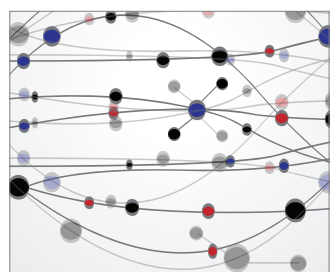

\section{The Scientific} World Journal
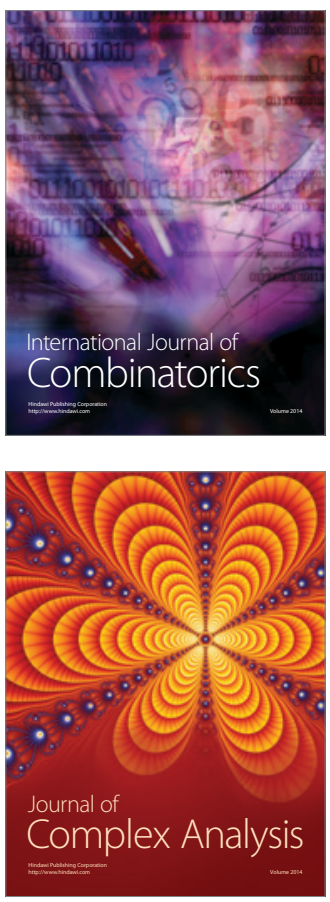

International Journal of

Mathematics and

Mathematical

Sciences
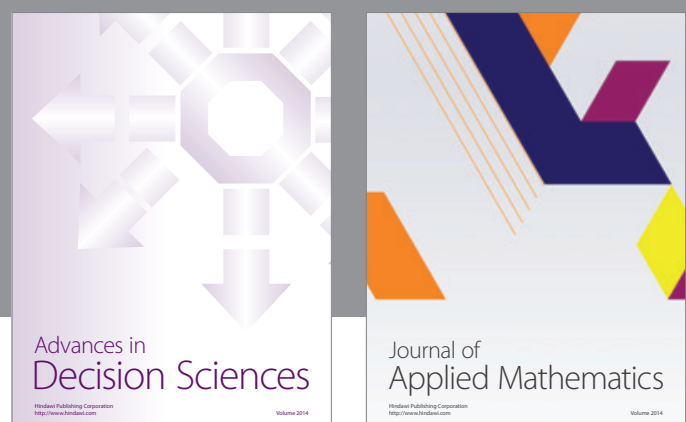

Journal of

Applied Mathematics
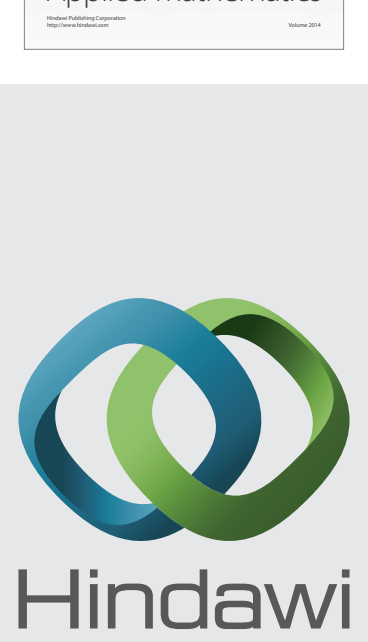

Submit your manuscripts at http://www.hindawi.com
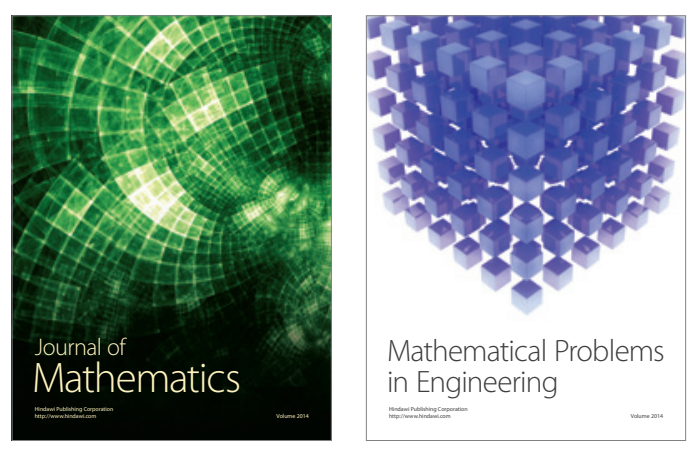

Mathematical Problems in Engineering
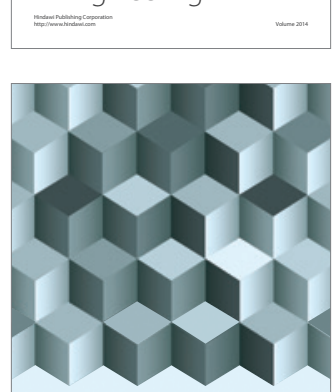

Journal of

Function Spaces
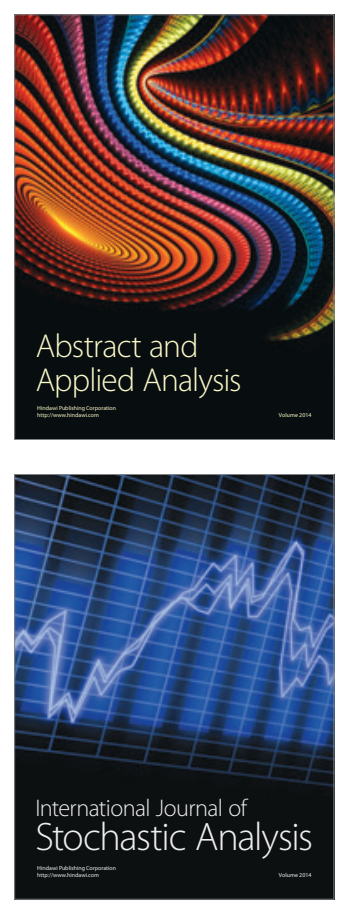

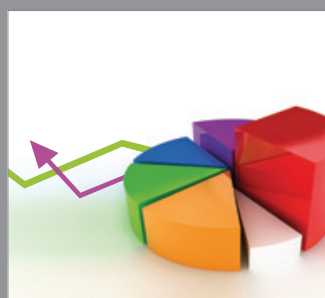

ournal of

Probability and Statistics

Promensencen
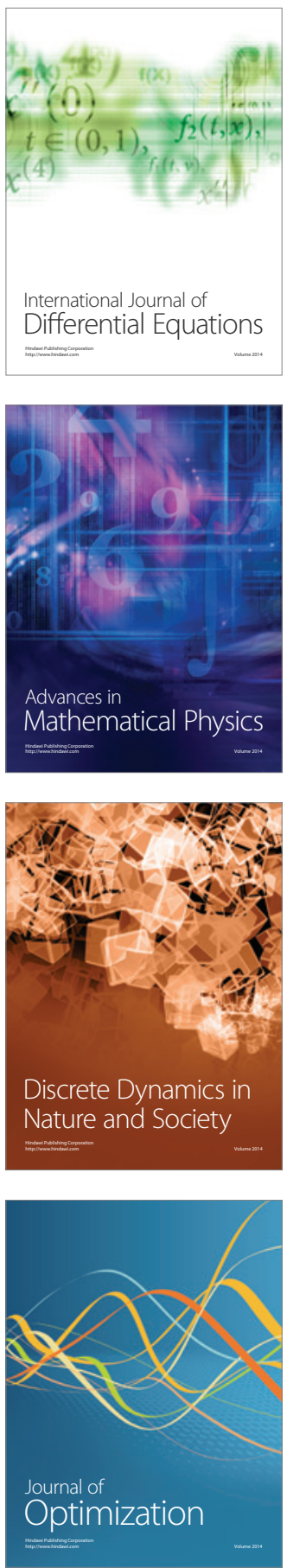